\title{
COMPORTAMIENTO PRODUCTIVO DE ASOCIACIONES DE GRAMÍNEAS CON LEGUMINOSAS EN PASTOREO
}

\section{PRODUCTIVE PERFORMANCE OF GRASS-LEGUME ASSOCIATIONS UNDER GRAZING CONDITIONS}

\author{
Rigoberto Castro Rivera ${ }^{1}$, Alfonso Hernández Garay ${ }^{2 \star}$, Humberto Vaquera Huerta ${ }^{1}$, J. de la Paz Hernández \\ Girón', Adrián R. Quero Carrillo², Javier F. Enríquez Quiroz ${ }^{3}$ y Pedro A. Martínez Hernández ${ }^{4}$
}

${ }^{1}$ Centro Interdiciplinario de Investigación para el Desarrollo Integral Regional, Instituto Politecnico Nacional, Unidad Oaxaca. Hornos 1003, Sta. Cruz Xoxocotlán, Oaxaca, México. Tel. 019515170610 Ext. 82754. ${ }^{2}$ Colegio de Postgraduados-Campus Montecillo. Km 36.5 carretera México-Texcoco. 56230, Montecillo, Texcoco, Estado de México. ${ }^{3}$ Campo Experimental Cotaxtla. Instituto Nacional de Investigaciones Forestales Agrícolas y Pecuarias. Álamos 29, Col. Limón de Guerrero, Isla, Veracruz, México ${ }^{4}$ Departamento de Zootecnia, Universidad Autónoma Chapingo. 56230, Chapingo, Estado de México.

Autor para correspondencia (hernan@colpos.mx)

\section{RESUMEN}

Se evaluaron varias asociaciones de dos gramíneas (ovillo, Dactylis glomerata; y ballico perenne, Lolium perenne) y una leguminosa (trébol blanco, Trifolium repens) en diferentes proporciones, para buscar la de máximo rendimiento de materia seca (MS). Se utilizaron cinco proporciones (\%) de trébol blanco, ovillo y ballico perenne: 40:30:30; 40:0:60; 40:60:0; 40:20:40 y 40:40:20, que se distribuyeron en 20 unidades experimentales bajo un diseño de bloques completos al azar con cuatro repeticiones. Las variables evaluadas fueron: rendimiento de materia seca, tasa de crecimiento, altura de planta y composición botánica. Las praderas fueron pastoreadas por ovinos de la cruza entre las razas Suffolk $x$ Dorset, cada cuatro semanas en primaveraverano y cada seis en otoño-invierno. La asociación 40:20:40 mostró el mayor rendimiento de MS que superó en $52 \%$ a la asociación 40:60:0 que produjo el valor más bajo $(\mathrm{P} \leq 0.05)$. En todas las asociaciones el mayor rendimiento de MS ocurrió en primavera-verano y el menor en otoño-invierno, con 65 y $35 \%$ del rendimiento anual. En promedio, trébol blanco, ballico perenne y pasto ovillo aportaron 51, 34 y $17 \%$ respectivamente, del rendimiento de MS. Las mejores asociaciones fueron 40:20:40 y 40:30:30. Del análisis de mezclas se obtuvo que la asociación que maximiza el rendimiento de MS fue 40, 23 y $37 \%$ de trébol blanco, ovillo y ballico perenne, respectivamente. Dicho valor se obtuvo con la ecuación: Rendimiento de MS $\left(\mathrm{kg} \mathrm{ha}^{-1}\right)=11906$ (proporción de pasto ovillo) +14913 (proporción de ballico perenne) + 12770 (proporción de pasto ovillo) $x$ (proporción de ballico perenne).

Palabras clave: Dactylis glomerata, Lolium perenne, Trifolium repens, praderas asociadas, rendimiento de forraje.

\section{SUMMARY}

With the aim to maximize dry matter (DM) yield, several associations of two grasses (orchard grass, Dactylis glomerata, and perennial ryegrass, Lolium perenne), and one legume (white clover, Trifolium repens) were studied. Five proportions (\%) of white clover, orchard grass and perennial ryegrass were utilized: 40:30:30; 40:0:60; 40:60:0; 40:20:40 and 40:40:20, and allocated in 20 experimental units, under a randomized block design with four replications. The measured variables were dry matter yield, growth rate, plant height, and botanical composition. Swards were grazed by sheep Suffolk x Dorset, every four weeks in spring-summer and every six weeks in autumn-winter. The 40:20:40 association showed the highest DM yield, wich was $52 \%$ higher than that of 40:60:0 with the lowest value $(P \leq 0.05)$. In all the associations, the highest DM yield was observed in spring-summer and the lowest in autumn-winter, with 65 and $35 \%$ of the annual yield. On average, white clover, orchard grass and perennial ryegrass contributed 51, 31 and $17 \%$ of the total DM yield, respectively. The best associations were 40:20:40 and 40:30:30. From the mixture analysis, the ideal proportion that maximizes DM yield was 40,23 , and $37 \%$ of white clover, orchard grass and perennial ryegrass, respectively. These values were obtained using the following regression equation: DM yield (kg $\left.\mathrm{ha}^{-1}\right)=11906$ (orchard grass proportion) +14913 (perennial ryegrass proportion) +12770 (orchard grass proportion) $\mathrm{x}$ (perennial ryegrass proportion).

Index words: Dactylis glomerata, Lolium perenne, Trifolium repens, associated swards, herbage yield.

\section{INTRODUCCIÓN}

El aprovechamiento adecuado de praderas, puras o mixtas, requiere conocer la distribución estacional del rendimiento, así como las especies forrajeras y su respuesta al corte o defoliación. La tasa de crecimiento de una especie forrajera es muy sensible a la temperatura ambiental y a la precipitación (McKenzie et al., 1999), lo cual determina el rendimiento de materia seca (MS) tanto estacional como anual (Matthew et al., 2001; Lemaire, 2001). La asociación de especies forrajeras también afecta al rendimiento total, distribución estacional y la contribución de cada una de las especies, por lo que es necesario conocer su proporción óptima en la pradera (Karsten y Carlassare, 2002).

Al evaluar cuatro variedades de alfalfa (Medicago sativa L.), asociadas con trébol blanco (Trifolium repens), ballico perenne (Lolium perenne), festuca alta (Festuca arundinacea) y pasto ovillo (Dactylis glomerata), Camacho 
y García (2002) encontraron que la mayor altura de la planta se registró en verano $(54 \mathrm{~cm})$ y la más baja en invierno $(33$ $\mathrm{cm}$ ); además observaron que la contribución de las especies al rendimiento de forraje varió con la estación del año: en primavera y verano las leguminosas aportaron $69 \%$, mientras que en otoño e invierno aportaron $61 \%$.

En praderas mixtas con más de dos especies, Sanderson et al. (2005) detectaron que en la época húmeda no hubo diferencias en producción de forraje entre asociaciones, con un promedio de $9800 \mathrm{~kg}$ de MS ha-1, pero durante la época de estiaje las praderas con dos especies asociadas produjeron menor cantidad de forraje que las asociaciones con más de seis especies (4800 vs. $7600 \mathrm{~kg}_{\text {de }} \mathrm{MS} \mathrm{ha}^{-1}$ ).

Con la asociación de gramíneas y leguminosas se mejora la fertilidad del suelo respecto a los monocultivos. Esto se debe al mayor aporte de nitrógeno atmosférico, mayor intercepción de luz y distribución estacional de biomasa más homogénea (Zaragoza et al., 2009). La ventaja de usar leguminosas es su mayor persistencia en condiciones de pastoreo (Quero et al., 2007). No obstante, el uso de la asociación gramínea-leguminosa es restringido debido al menor crecimiento de cada especie, a la baja apetencia por los animales domésticos, a la renuencia y desconocimiento de los productores y a la escasa disponibilidad de semilla (Karsten y Carlassare, 2002).

En México los patrones de producción de forrajes están influenciados por variaciones del clima, por lo que es importante conocer los patrones estacionales de crecimiento de las especies forrajeras más utilizadas en cada región ecológica. En asociaciones de gramíneas y leguminosas es necesario conocer la mejor combinación, desde el punto de vista de rendimiento, distribución estacional y persistencia de la pradera.

Por lo anterior, el objetivo del presente estudio fue evaluar el rendimiento anual y estacional, tasa de crecimiento, altura de planta y composición botánica de la asociación de trébol blanco, pasto ovillo y ballico perenne, mediante un modelo de análisis de mezclas que permite determinar la combinación óptima que maximiza el rendimiento.

\section{MATERIALES Y MÉTODOS}

El estudio se llevó a cabo en una pradera mixta de trébol blanco, pasto ovillo y ballico perenne, establecida en Marzo del 2006 en Montecillo, Texcoco, Estado de México (19³1' LN y $\left.98^{\circ} 53^{\prime} \mathrm{LO}\right)$. El suelo del sitio es franco arenoso, ligeramente alcalino ( $\mathrm{pH} 7.8$ ), con $2.4 \%$ de materia orgánica y se clasifica como Typic ustipsaments (Ortiz, 1997). El clima es templado subhúmedo con lluvias en verano, precipitación media anual de $645 \mathrm{~mm}$ y temperatura media anual de $15^{\circ} \mathrm{C}$; la temperatura promedio mensual más baja es de $11.6^{\circ} \mathrm{C}$ y ocurre en enero y la más alta en mayo con $18.4^{\circ} \mathrm{C}$ (García, 1988).

Las gramíneas se sembraron en hileras separadas a $30 \mathrm{~cm}$, y la leguminosa en forma perpendicular a las gramíneas, con hileras a $40 \mathrm{~cm}$. Se utilizaron cinco proporciones (\%) de trébol blanco, ovillo y ballico perenne: 40:30:30; 40:0:60; 40:60:0; 40:20:40 y 40:40:20, que se distribuyeron en 20 unidades experimentales de $13 \times 8 \mathrm{~m}$, distribuidas en un diseño de bloques completos al azar con cuatro repeticiones. Los bloques fueron orientados perpendicularmente a la pendiente del terreno. Las unidades experimentales no fueron fertilizadas, y en la época de estiaje se aplicaron riegos a capacidad de campo cada dos semanas. El experimento se inició en Marzo de 2007.

$\mathrm{Al}$ inicio del experimento las praderas fueron pastoreadas por ovinos (agregar raza y edad del rebaño) para uniformizarlas a $5 \mathrm{~cm}$ sobre el nivel del suelo. Posteriormente, la frecuencia de pastoreo fue cada cuatro semanas en primavera-verano y cada seis semanas en otoño-invierno, de acuerdo con las recomendaciones de Velasco et al. (2001, 2005), para pasto ovillo y ballico perenne en monocultivo. Para un mejor manejo de los ovinos, las praderas fueron delimitadas con cerco eléctrico.

\section{Variables estudiadas}

Rendimiento de forraje. El rendimiento de forraje de cada parcela se midió en dos cuadros fijos de $0.25 \mathrm{~m}^{2}$ en los que se cortó el forraje con tijeras antes del pastoreo, a $5 \mathrm{~cm}$ de altura. Inmediatamente después, las parcelas fueron pastoreadas por ovinos hasta dejarlas a una altura de $5 \mathrm{~cm}$, aproximadamente. El material cosechado se lavó y secó en una estufa de aire forzado a $55^{\circ} \mathrm{C}$ por $48 \mathrm{~h}$ para alcanzar un peso constante. Con estos datos se calcularon los rendimientos estacional y anual para cada asociación.

Tasa de crecimiento del cultivo (TC). Con los datos de rendimiento de materia seca (MS) por corte se calculó la tasa de crecimiento para cada asociación, mediante la siguiente fórmula:

$$
T C=F C / t
$$

Dónde: $F C=$ Forraje cosechado $(\mathrm{kg} \mathrm{MS} \mathrm{ha-1}), \mathrm{y} t=$ días transcurridos entre un corte y el siguiente.

Altura de planta. Se midió antes de cada pastoreo con una regla de $1 \mathrm{~m}$ de longitud y graduación mínima de 1 $\mathrm{mm}$. Se eligieron aleatoriamente 20 puntos por parcela, y la regla se aproximó verticalmente hacia el tejido vegetal más alto, para registrar la altura de la primera especie 
contactada (Hodgson et al., 1999).

Composición botánica. A la mitad de cada estación, de las muestras cosechadas para medir el rendimiento de MS se tomó $20 \%$ y las plantas se clasificaron por especie en estudio, material muerto, otros pastos y maleza. Las muestras se secaron a $55^{\circ} \mathrm{C}$ por $48 \mathrm{~h}$ hasta peso constante, y luego se pesaron.

Análisis de mezclas. Se usó el modelo para un diseño de un experimento de mezclas con dos factores de estudio: $Y=b_{1} X_{1}+b_{2} X_{2}+b_{3} X_{1} X_{2}+e$, sujeto a la restricción $X_{1}+X_{2}$ $=1$ donde $X_{1}$ representa la proporción de pasto ovillo y $X_{2}$ representa la proporción de ballico perenne, $Y$ es el rendimiento de materia seca por hectárea, y $\boldsymbol{e}$ es el error aleatorio. Con el paquete estadístico MINITAB (2006) se estimaron las constantes $b_{1}, b_{2} y b_{3}$ y resultó la siguiente ecuación de regresión: Rendimiento de MS $\left(\mathrm{kg} \mathrm{ha}^{-1}\right)=11$ $906 X_{1}+14913 X_{2}+12770 X_{1} X_{2}$

Datos climáticos. Los promedios mensuales de temperatura a la intemperie (máxima, media y mínima) y precipitación mensual durante el periodo de estudio se obtuvieron de una estación climatológica situada a $100 \mathrm{~m}$ del sitio experimental.

Análisis estadístico. Los valores de cada variable se organizaron de manera estacional y anual, y con ello se hicieron comparaciones de medias $(\mathrm{P} \leq 0.05)$ mediante el procedimiento PROCMIXED (SAS Institute, 2002). También se estimó la combinación que maximiza el rendimiento de materia seca (MINITAB, 2006).

\section{RESULTADOS Y DISCUSIÓN}

\section{Rendimiento de forraje}

Con excepción de la asociación 40:60:0 que registró el menor rendimiento anual de materia seca, no hubo diferencias entre tratamientos (Cuadro 1). El rendimiento anual de las asociaciones de trébol blanco, ovillo y ballico perenne mostró el siguiente orden descendente: 40:20:40 $>$ 40:30:30 > 40:0:60 > 40:40:20 > 40:60:0. La asociación 40:20:40 superó en $52 \%$ a la asociación 40:60:0 que fue la que obtuvo el valor más bajo $(\mathrm{P}<0.05)$. Las asociaciones 40:20:40 y 40:30:30 que registraron los valores más altos de rendimiento de materia seca, tuvieron un aporte de 47 y 49 $\%$ para el trébol blanco, seguido del ballico perenne con 23 y $27 \%$, y del ovillo con 9 y $12 \%$.

Los resultados (Cuadro 1) a través de estaciones muestran que $65 \%$ del rendimiento anual se presentó en primavera y verano, y $35 \%$ en otoño e invierno; otoño fue la estación que presentó el menor valor, con $12 \%(\mathrm{P} \leq 0.05)$. En primavera y verano el mayor rendimiento lo mostró la asociación 40:20:40 con 30 y $35 \%$ del rendimiento anual, y sólo fue estadísticamente superior a la asociación 40:60:0 $(\mathrm{P} \leq 0.05)$. Sin embargo, en otoño e invierno la asociación 40:30:30 fue la que registró el mayor rendimiento, aunque solamente superó $(\mathrm{P} \leq 0.05)$ a la asociación 40:60:0.

Independientemente de la asociación, el rendimiento de materia seca fue de $29,35,12$ y $23 \%$ para primavera, verano, otoño e invierno, respectivamente; los valores más altos se atribuyen a que en primavera-verano se registraron las condiciones ambientales más favorables, particularmente de temperatura, lo que permitió a las especies manifestar un elevado potencial productivo (Figura 1). Al respecto, se ha señalado que una mayor temperatura aumenta la tasa de aparición y expansión foliar que combinada con un nivel adecuado de humedad, hace que la pradera alcance rápidamente su índice de área foliar óptimo en primavera y verano, como consignaron Velasco et al. $(2001,2002)$ para

Cuadro 1. Rendimiento estacional y anual de forraje $\left(\mathrm{kg} \mathrm{MS} \mathrm{ha}^{-1}\right)$ de cinco asociaciones de gramíneas con una leguminosa.

\begin{tabular}{|c|c|c|c|c|c|c|c|c|}
\hline \multirow{2}{*}{$\begin{array}{l}\text { Estación del } \\
\text { año }\end{array}$} & \multicolumn{5}{|c|}{ Asociaciones (trébol blanco: ovillo: ballico perenne; \%) } & \multirow{2}{*}{ EEM } & \multirow{2}{*}{ Sig. } & \multirow{2}{*}{ Prom } \\
\hline & $40: 30: 30$ & 40:0:60 & 40:60:0 & $40: 20: 40$ & $40: 40: 20$ & & & \\
\hline Primavera & $4514 \mathrm{ABb}$ & $4813 \mathrm{ABa}$ & $3330 \mathrm{Bab}$ & $5137 \mathrm{Ab}$ & $4434 \mathrm{ABb}$ & 493 & * & $4446 \mathrm{~b}$ \\
\hline Verano & $5803 \mathrm{Aa}$ & $5464 \mathrm{Aa}$ & $4239 \mathrm{Ba}$ & $6096 \mathrm{Aa}$ & $5329 \mathrm{ABa}$ & 370 & * & $5386 \mathrm{a}$ \\
\hline Otoño & $2319 \mathrm{Ac}$ & $1863 \mathrm{ABc}$ & $1336 \mathrm{Bc}$ & $2088 \mathrm{Ad}$ & $1725 \mathrm{ABd}$ & 237 & * & $1866 \mathrm{~d}$ \\
\hline Invierno & $4149 \mathrm{Ab}$ & $3347 \mathrm{ABb}$ & $2452 \mathrm{Cbc}$ & $3954 \mathrm{ABC}$ & $3498 \mathrm{ABc}$ & 404 & * & $3480 \mathrm{c}$ \\
\hline Promedio & $4196 \mathrm{AB}$ & $3872 \mathrm{AB}$ & $2839 \mathrm{C}$ & $4318 \mathrm{~A}$ & $3746 \mathrm{~B}$ & 195 & $* *$ & \\
\hline EEM & 271 & 296 & 34 & 242 & 206 & & & 174 \\
\hline Sig. & ** & $* *$ & $* *$ & $* *$ & ** & & & $* *$ \\
\hline Rend. anual & $16785 \mathrm{~A}$ & 15487 A & 11357 B & $17275 \mathrm{~A}$ & $14986 \mathrm{~A}$ & 953 & * & \\
\hline
\end{tabular}

Medias con letras mayúsculas iguales en cada hilera no son estadísticamente diferentes, y medias con letras minúsculas iguales en cada columna no son estadísticamente diferentes (Tukey, 0.05). EEM = error estándar de la media; Sig. $=$ significancia; ${ }^{*}=\mathrm{P}<0.05$; ${ }^{* *}=\mathrm{P}<0.01 ;$ Prom. $=$ promedio. 


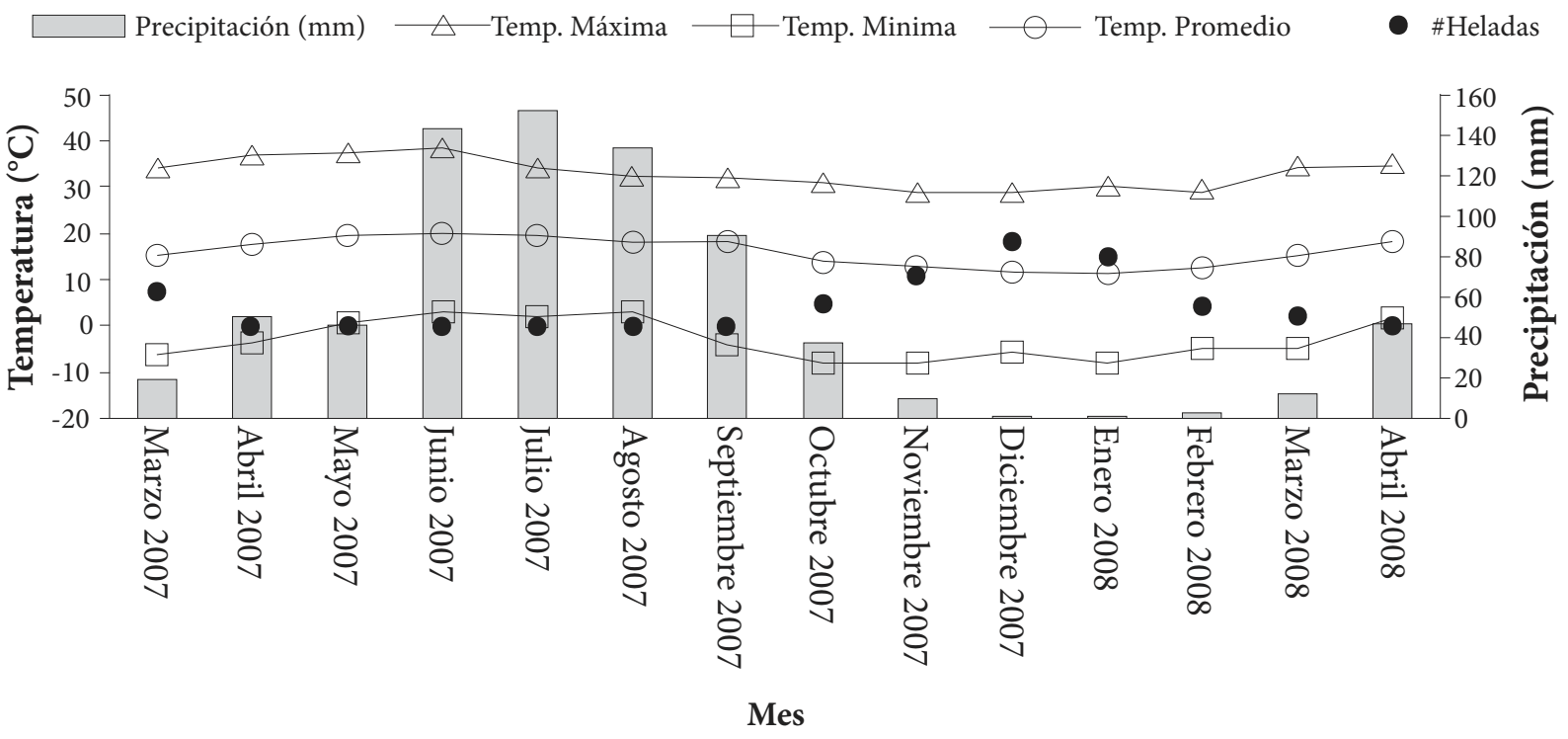

Figura 1. Datos de clima durante el periodo experimental. Fuente: Estación meteorológica del Colegio de Postgraduados en Montecillo, Texcoco, Estado de México.

pasto ovillo y ballico perenne, así como Clark et al. (1995) y Brock et al. (1989) para trébol blanco.

Al comparar la contribución de cada especie al rendimiento anual (Cuadro 2), en todas las asociaciones el trébol blanco aportó más de $50 \%$ del rendimiento, que durante la época de primavera-verano subió a $65 \%$ del rendimiento anual, mientras que en otoño aportó apenas $12 \%$. La asociación 40:20:40 con 10066 kg MS ha-1 fue la que presentó el mayor rendimiento anual de trébol blanco, y que superó en $96 \%$ a 40:60:0 ( $\mathrm{P} \leq 0.05)$. Al respecto, Hodgson (1990) señaló que después de una defoliación el restablecimiento del rebrote del trébol blanco resulta del equilibrio entre la luz y la temperatura, y el número y tamaño de las hojas presentes. Es de suponer que el arreglo horizontal de los foliolos del trébol blanco ayuda a restablecer su área foliar más rápidamente que el ballico, pero conforme el intervalo de rebrote aumenta, esta ventaja favorece a las hojas erectas del ballico que sombrean a las del trébol blanco.

El pasto ovillo fue la especie que registró el menor rendimiento en las asociaciones, con un promedio anual de $2560 \mathrm{~kg} \mathrm{MS} \mathrm{ha-1} \mathrm{(Cuadro} \mathrm{2).} \mathrm{En} \mathrm{verano} \mathrm{registró} \mathrm{el} \mathrm{mayor}$ rendimiento con $40 \%$ del total $(\mathrm{P} \leq 0.05)$. Las asociaciones 40:20:40 y 40:60:0, con 4420 y $1540 \mathrm{~kg} \mathrm{MS} \mathrm{ha}^{-1}$, fueron las que presentaron el menor y mayor rendimiento de pasto ovillo ( $\mathrm{P} \leq 0.05)$. La escasa contribución del ovillo al rendimiento anual pudo deberse a que cuando esta especie es sometida a una severa intensidad de pastoreo y queda con un mínimo de hojas remanentes, el rebrote es lento; por ello se recomienda dejar tres hojas por tallo, para asegurar un rebrote vigoroso (Turner et al., 2006).

La contribución del ballico perenne al rendimiento anual fue en promedio de $4670 \mathrm{~kg} \mathrm{MS} \mathrm{ha}^{-1}$ y la asociación 40:0:60 fue la que presentó el mejor rendimiento con 6012 $\mathrm{kg} \mathrm{MS} \mathrm{ha-1,} \mathrm{superior} \mathrm{a} \mathrm{la} \mathrm{asociación} \mathrm{40:40:20} \mathrm{que} \mathrm{tuvo} \mathrm{el}$ menor rendimiento de ballico con $4011 \mathrm{~kg} \mathrm{MS} \mathrm{ha}^{-1}(\mathrm{P} \leq$ 0.05). Durante el otoño se registró el menor rendimiento promedio de ballico perenne con $611 \mathrm{~kg}$ de $\mathrm{MS} \mathrm{ha}^{-1}(\mathrm{P} \leq$ $0.05)$, pero sin diferencias entre las demás estaciones del año.

La mayor aportación del trébol blanco al rendimiento anual, particularmente en las épocas de primavera y verano, se atribuye a que la temperatura ambiental fue cercana a la óptima para el crecimiento de esta especie, que es de $24^{\circ} \mathrm{C}$ (Brock y Tilbrook, 2000), mientras que para ballico perenne y ovillo las óptimas son de 18 a $21^{\circ} \mathrm{C}$, por lo que estuvieron en desventaja con el trébol blanco. En otoño se registró el menor rendimiento de las tres especies evaluadas, debido a que se presentaron las temperaturas mínimas más bajas (Figura 1). Es conocido que las bajas temperaturas, aun por periodos cortos, causan reducción del crecimiento de los pastos y de sus tasas de acumulación de forraje (Hernández et al., 1997).

\section{Tasa de crecimiento}

La tasa de crecimiento (TC) estacional de las cinco asociaciones se presenta en el Cuadro 3. La asociación 40:60:0 con $32 \mathrm{~kg} \mathrm{MS} \mathrm{ha}^{-1} \mathrm{~d}^{-1}$ presentó el menor promedio anual ( $\mathrm{P} \leq 0.05)$, que fue 59, 53, 37 y $34 \%$ inferior al de 
Cuadro 2. Rendimiento de forraje estacional y anual ( $\mathrm{kg}$ MS ha ${ }^{-1}$ ) por especie deseable de cinco asociaciones de gramíneas con una leguminosa.

\begin{tabular}{|c|c|c|c|c|c|c|c|c|}
\hline \multirow{2}{*}{$\begin{array}{l}\text { Estación del } \\
\text { año }\end{array}$} & \multicolumn{5}{|c|}{ Asociaciones (trébol blanco: ovillo: ballico perenne; \%) } & \multirow{2}{*}{ EEM } & \multirow{2}{*}{ Sig. } & \multirow{2}{*}{ Prom. } \\
\hline & $40: 30: 30$ & 40:0:60 & 40:60:0 & $40: 20: 40$ & $40: 40: 20$ & & & \\
\hline \multicolumn{9}{|c|}{ Trébol blanco (kg MS ha') } \\
\hline Primavera & $2167 \mathrm{ABab}$ & $2529 \mathrm{ABa}$ & $1272 \mathrm{Bab}$ & $3451 \mathrm{Aa}$ & $2191 \mathrm{ABa}$ & 639 & ** & $2322 \mathrm{a}$ \\
\hline Verano & $2885 \mathrm{ABa}$ & $2692 \mathrm{ABa}$ & $1797 \mathrm{Ba}$ & $3289 \mathrm{Aa}$ & $2536 \mathrm{ABa}$ & 427 & ** & $2640 \mathrm{a}$ \\
\hline Otoño & $1214 \mathrm{Ab}$ & $884 \mathrm{ABb}$ & $534 \mathrm{Bb}$ & $1195 \mathrm{Ab}$ & $742 \mathrm{ABb}$ & 190 & * & $914 \mathrm{c}$ \\
\hline Invierno & $2012 \mathrm{ab}$ & $1465 \mathrm{ab}$ & $1527 \mathrm{ab}$ & $2130 \mathrm{ab}$ & $1681 \mathrm{ab}$ & 439 & NS & $1763 \mathrm{~b}$ \\
\hline Promedio & $2070 \mathrm{ab}$ & $1892 \mathrm{~b}$ & $1282 \mathrm{c}$ & $2116 \mathrm{a}$ & $1787 \mathrm{bc}$ & 219 & $* *$ & \\
\hline EEM & 411 & 476 & 395 & 517 & 345 & & & 195 \\
\hline Sig. & ** & ** & * & ** & $* *$ & & & ** \\
\hline Rend. anual & $8278 \mathrm{AB}$ & $7570 \mathrm{ABC}$ & $5129 \mathrm{C}$ & $10066 \mathrm{~A}$ & $7150 \mathrm{BC}$ & 984 & * & \\
\hline \multicolumn{9}{|c|}{ Pasto Ovillo (kg MS ha' $\left.{ }^{-1}\right)$} \\
\hline Primavera & $435 \mathrm{Bb}$ & - & $1533 \mathrm{Aa}$ & $354 \mathrm{Bab}$ & $697 \mathrm{Ba}$ & 151 & ** & $755 \mathrm{~b}$ \\
\hline Verano & $924 \mathrm{Ba}$ & - & $1594 \mathrm{Aa}$ & $585 \mathrm{Ba}$ & $945 \mathrm{Ba}$ & 196 & ** & $1012 \mathrm{a}$ \\
\hline Otoño & $298 \mathrm{Bb}$ & - & $621 \mathrm{Ab}$ & $153 \mathrm{Bb}$ & $268 \mathrm{Bb}$ & 90 & ** & $335 c$ \\
\hline Invierno & $430 \mathrm{ABb}$ & - & $671 \mathrm{Ab}$ & $448 \mathrm{ABa}$ & $278 \mathrm{Bb}$ & 93 & ** & $457 \mathrm{c}$ \\
\hline Promedio & $522 \mathrm{~B}$ & - & $1105 \mathrm{~A}$ & $385 \mathrm{C}$ & $547 \mathrm{~B}$ & 69 & ** & \\
\hline EEM & 121 & & 226 & 107 & 127 & & & 62 \\
\hline Sig. & $* *$ & & $* *$ & $* *$ & ** & & & $* *$ \\
\hline Rend. anual & 2086 B & - & $4419 \mathrm{~A}$ & $1541 \mathrm{~B}$ & $2188 \mathrm{~B}$ & 385 & ** & \\
\hline \multicolumn{9}{|c|}{ Ballico perenne (kg MS ha-1) } \\
\hline Primavera & $1506 \mathrm{Aa}$ & $1852 \mathrm{Aa}$ & - & $864 \mathrm{~B} a b$ & $1040 \mathrm{ABab}$ & 368 & * & $1316 \mathrm{a}$ \\
\hline Verano & $1240 \mathrm{ab}$ & $1825 \mathrm{a}$ & - & $1595 \mathrm{a}$ & $1230 \mathrm{a}$ & 266 & NS & $1472 \mathrm{a}$ \\
\hline Otoño & $594 \mathrm{~b}$ & $788 \mathrm{~b}$ & - & $535 \mathrm{~b}$ & $525 \mathrm{~b}$ & 126 & NS & $611 \mathrm{~b}$ \\
\hline Invierno & $1296 \mathrm{a}$ & $1547 \mathrm{ab}$ & - & $1025 \mathrm{ab}$ & $1216 \mathrm{a}$ & 201 & NS & $1270 \mathrm{a}$ \\
\hline Promedio & $1159 \mathrm{AB}$ & $1503 \mathrm{~A}$ & - & $1005 \mathrm{~B}$ & $1003 \mathrm{~B}$ & 129 & $* *$ & \\
\hline EEM & 244 & 326 & & 331 & 199 & & & 116 \\
\hline Sig. & $* *$ & $* *$ & & $* *$ & $* *$ & & & $* *$ \\
\hline Rend. anual & $4636 \mathrm{AB}$ & $6012 \mathrm{~A}$ & - & 4018 B & $4011 \mathrm{~B}$ & 601.77 & ** & \\
\hline
\end{tabular}

Cuadro 3. Tasa de crecimiento ( $\mathrm{kg} \mathrm{MS} \mathrm{ha-1} \mathrm{d}^{-1}$ ) promedio estacional y anual de cinco asociaciones de gramíneas con una leguminosa.

\begin{tabular}{|c|c|c|c|c|c|c|c|c|}
\hline \multirow{2}{*}{$\begin{array}{l}\text { Estación del } \\
\text { año }\end{array}$} & \multicolumn{5}{|c|}{ Asociaciones (trébol blanco: ovillo: ballico perenne; \%) } & \multirow{2}{*}{ EEM } & \multirow{2}{*}{ Sig. } & \multirow{2}{*}{ Prom } \\
\hline & $40: 30: 30$ & 40:0:60 & 40:60:0 & 40:20:40 & 40:40:20 & & & \\
\hline Primavera & $54 \mathrm{ABa}$ & $57 \mathrm{Aab}$ & $40 \mathrm{Bab}$ & $61 \mathrm{Aab}$ & $53 \mathrm{ABab}$ & 6 & * & $50 \mathrm{~b}$ \\
\hline Verano & $69 \mathrm{Aa}$ & $65 \mathrm{Aa}$ & $50 \mathrm{Ba}$ & $73 \mathrm{Aa}$ & $63 \mathrm{Aa}$ & 4 & ** & $63 \mathrm{a}$ \\
\hline Otoño & $28 \mathrm{Ab}$ & $22 \mathrm{ABc}$ & $16 \mathrm{Bc}$ & $25 \mathrm{Ac}$ & $21 \mathrm{ABc}$ & 3 & ** & $20 \mathrm{~d}$ \\
\hline Invierno & $49 \mathrm{Aa}$ & $40 \mathrm{ABb}$ & $29 \mathrm{Bbc}$ & $47 \mathrm{Ab}$ & $42 \mathrm{ABb}$ & 5 & $* *$ & $41 \mathrm{c}$ \\
\hline Promedio & $51 \mathrm{~A}$ & $43 \mathrm{~A}$ & $32 \mathrm{~B}$ & $49 \mathrm{~A}$ & $44 \mathrm{~A}$ & 3 & $* *$ & \\
\hline EEM & 7 & 6 & 6 & 6 & 6 & & & 3 \\
\hline Sig. & $* *$ & $* *$ & $* *$ & $* *$ & $* *$ & & & $* *$ \\
\hline
\end{tabular}


Cuadro 4. Tasa de crecimiento promedio estacional y anual (kg MS ha-1 $\mathrm{d}^{-1}$ ) por especie, en de cinco asociaciones de gramíneas con una leguminosa.

\begin{tabular}{|c|c|c|c|c|c|c|c|c|}
\hline \multirow{2}{*}{$\begin{array}{l}\text { Estación del } \\
\text { año }\end{array}$} & \multicolumn{5}{|c|}{ Asociaciones (trébol blanco: ovillo: ballico perenne; \%) } & \multirow{2}{*}{ EEM } & \multirow{2}{*}{ Sig. } & \multirow{2}{*}{ Prom. } \\
\hline & $40: 30: 30$ & $40: 0: 60$ & 40:60:0 & $40: 20: 40$ & $40: 40: 20$ & & & \\
\hline \multicolumn{9}{|c|}{ Trébol blanco (kg MS ha $\left.{ }^{-1} \mathrm{~d}^{-1}\right)$} \\
\hline Primavera & $26 \mathrm{ABab}$ & $30 \mathrm{ABa}$ & $15 \mathrm{Bab}$ & $41 \mathrm{Aa}$ & $26 \mathrm{ABa}$ & 8 & ** & $28 \mathrm{a}$ \\
\hline Verano & $34 \mathrm{ABa}$ & $32 \mathrm{ABa}$ & $21 \mathrm{Ba}$ & $39 \mathrm{Aa}$ & $30 \mathrm{ABa}$ & 5 & ** & $31 \mathrm{a}$ \\
\hline Otoño & $15 \mathrm{Ab}$ & $11 \mathrm{ABb}$ & $6 \mathrm{Bb}$ & $14 \mathrm{Ab}$ & $9 \mathrm{ABb}$ & 2 & $* *$ & $11 \mathrm{c}$ \\
\hline Invierno & $24 \mathrm{ab}$ & $17 \mathrm{ab}$ & $18 \mathrm{ab}$ & $25 \mathrm{ab}$ & $20 \mathrm{ab}$ & 4 & NS & $21 b$ \\
\hline Promedio & $25 \mathrm{AB}$ & $23 \mathrm{~B}$ & $15 \mathrm{C}$ & $30 \mathrm{~A}$ & $21 \mathrm{BC}$ & 3 & ** & \\
\hline EEM & 5 & 6 & 5 & 6 & 4 & & & 2 \\
\hline Sig. & ** & $* *$ & ** & $* *$ & $* *$ & & & ** \\
\hline \multicolumn{9}{|c|}{ Pasto Ovillo (kg MS ha-1 $\mathrm{d}^{-1}$ ) } \\
\hline Primavera & $5 \mathrm{Bb}$ & - & $18 \mathrm{Aa}$ & $5 \mathrm{Bab}$ & $8 \mathrm{Ba}$ & 2 & ** & $7 \mathrm{~b}$ \\
\hline Verano & $11 \mathrm{Ba}$ & - & $19 \mathrm{Aa}$ & $7 \mathrm{Ba}$ & $11 \mathrm{Ba}$ & 2 & ** & $10 \mathrm{a}$ \\
\hline Otoño & $4 \mathrm{Bb}$ & - & $7 \mathrm{Ab}$ & $2 \mathrm{Bb}$ & $3 \mathrm{Bb}$ & 1 & ** & $3 c$ \\
\hline Invierno & $5 \mathrm{ABb}$ & - & $8 \mathrm{Ab}$ & $5 \mathrm{ABa}$ & $3 \mathrm{Bb}$ & 1 & ** & $4 \mathrm{c}$ \\
\hline Promedio & $6 \mathrm{~B}$ & & $13 \mathrm{~A}$ & $5 \mathrm{~B}$ & $7 \mathrm{~B}$ & 1 & ** & \\
\hline EEM & 1 & & 3 & 1 & 2 & & & 1 \\
\hline Sig. & $* *$ & & ** & $* *$ & $* *$ & & & ** \\
\hline \multicolumn{9}{|c|}{ Ballico perenne (kg MS ha-1 $\left.\mathrm{d}^{-1}\right)$} \\
\hline Primavera & $18 \mathrm{a}$ & $22 \mathrm{a}$ & - & $10 \mathrm{ab}$ & $12 \mathrm{ab}$ & 4 & NS & $13 \mathrm{a}$ \\
\hline Verano & $15 \mathrm{ab}$ & $22 \mathrm{a}$ & - & $19 \mathrm{a}$ & $15 \mathrm{a}$ & 3 & NS & $14 \mathrm{a}$ \\
\hline Otoño & $7 \mathrm{~b}$ & $9 \mathrm{~b}$ & - & $6 \mathrm{~b}$ & $6 \mathrm{~b}$ & 2 & NS & $6 \mathrm{~b}$ \\
\hline Invierno & $15 \mathrm{ab}$ & $18 \mathrm{ab}$ & - & $12 \mathrm{ab}$ & $14 \mathrm{ab}$ & 2 & NS & $12 \mathrm{a}$ \\
\hline Promedio & $14 \mathrm{AB}$ & $18 \mathrm{~A}$ & & $12 \mathrm{~B}$ & $12 \mathrm{~B}$ & 2 & $* *$ & \\
\hline EEM & 3 & 4 & & 4 & 2 & & & 1 \\
\hline Sig. & $* *$ & $* *$ & & $* *$ & $* *$ & & & ** \\
\hline
\end{tabular}

Medias con letras mayúsculas iguales en cada hilera no son estadísticamente diferentes y medias con letras minúsculas iguales en cada columna no son estadísticamente diferentes (Tukey, 0.05). EEM = error estándar de la media; Sig.= significancia; ${ }^{*}=\mathrm{P}$ $<0.05 ;{ }^{* *}=\mathrm{P}<0.01 ; \mathrm{NS}=$ no significativo; Prom. $=$ promedio.

las asociaciones 40:30:30, 40:20:40, 40:40:20 y 40:0:60, respectivamente, las cuales no fueron estadísticamente diferentes entre sí $(\mathrm{P} \leq 0.05)$. En general, la mayor TC estacional se observó durante el verano con $64 \mathrm{~kg} \mathrm{MS} \mathrm{ha}^{-1}$ $\mathrm{d}^{-1}$ y superó en 21, 55 y $186 \%$ a las de primavera, invierno y otoño, respectivamente. Durante el verano, la menor y mayor TC ocurrieron en las asociaciones 40:60:0 y 40:20:40 (P $\leq$ $0.05)$, con 50 y $73 \mathrm{~kg} \mathrm{MS} \mathrm{ha}^{-1} \mathrm{~d}^{-1}$. En primavera, la asociación 40:20:40 superó en $7,13,15$ y $53 \%$ a las asociaciones 40:0:60, 40:30:30, 40:40:20 y 40:60:0, respectivamente, pero sólo esta última fue diferente $(\mathrm{P} \leq 0.05)$ a la primera. Estos resultados coinciden con los observados por Velasco et al. (2001, 2002) quienes en praderas puras de ovillo y ballico perenne registraron las mayores TC durante el verano.

En el Cuadro 4 se presentan las TC estacional y promedio anual de cada especie. Para el trébol blanco la TC promedio anual en la asociación 40:60:0 con $15 \mathrm{~kg}$ MS ha-1 $\mathrm{d}^{-1}$ fue $98,65,53$ y $42 \%$ inferior que en las asociaciones 40:20:40, 40:30:30, 40:0:60 y 40:40:20, respectivamente. En la época de verano, la TC del trébol blanco mostró el valor más alto (31 kg MS ha-1 $\mathrm{d}^{-1}$ ), y superó en 13, 50 y $184 \%$ a las de primavera, invierno y otoño, respectivamente $(\mathrm{P} \leq$ 0.05). La TC de la asociación 40:20:40 del verano superó en 86 ( $\mathrm{P} \leq 0.05), 15,22$ y $50 \%$ a las asociaciones 40:60:0, 40:30:30, 40:0:60 y 40:40:20. Las menores TC observadas en otoño estuvieron asociadas con el mayor número de heladas incidentes en la zona (Figura 1). Las mayores TC del trébol blanco en verano, fueron similares o superiores a las reportadas por Sevilla et al. (2001) en praderas puras de trébol blanco.

La mayor TC anual del pasto ovillo (Cuadro 4) se registró en la asociación 40:60:0 con $13 \mathrm{~kg} \mathrm{MS} \mathrm{ha-1} \mathrm{d}^{-1}$, y superó en 
Cuadro 5. Altura (cm) promedio anual y estacional de las plantas de cinco asociaciones de gramíneas con una leguminosa.

\begin{tabular}{|c|c|c|c|c|c|c|c|c|}
\hline \multirow{2}{*}{$\begin{array}{l}\text { Estación del } \\
\text { año }\end{array}$} & \multicolumn{5}{|c|}{ Asociaciones (trébol blanco: ovillo: ballico perenne; \%) } & \multirow{2}{*}{ EEM } & \multirow{2}{*}{ Sig. } & \multirow{2}{*}{ Prom. } \\
\hline & $40: 30: 30$ & 40:0:60 & 40:60:0 & $40: 20: 40$ & $40: 40: 20$ & & & \\
\hline Primavera & $23 \mathrm{Ab}$ & $22 \mathrm{Ab}$ & $18 \mathrm{Bb}$ & $23 \mathrm{Ab}$ & $23 \mathrm{Ab}$ & 0.5 & $* *$ & $22 \mathrm{~b}$ \\
\hline Verano & $28 \mathrm{Aa}$ & $26 \mathrm{BCa}$ & $22 \mathrm{Da}$ & $25 \mathrm{Ca}$ & $27 \mathrm{ABa}$ & 0.4 & $* *$ & $26 \mathrm{a}$ \\
\hline Otoño & $12 \mathrm{Ac}$ & $11 \mathrm{Ac}$ & $9 \mathrm{Bc}$ & $10 \mathrm{Bc}$ & $11 \mathrm{Ac}$ & 0.3 & $* *$ & $11 \mathrm{c}$ \\
\hline Invierno & $12 \mathrm{Ac}$ & $12 \mathrm{ABC}$ & $8 \mathrm{Cd}$ & $11 \mathrm{Bc}$ & $11 \mathrm{Bc}$ & 0.4 & $* *$ & $11 \mathrm{c}$ \\
\hline Promedio & $20 \mathrm{~A}$ & $19 \mathrm{~B}$ & $16 \mathrm{D}$ & $18 \mathrm{C}$ & $19 \mathrm{~B}$ & 0.21 & $* *$ & \\
\hline EEM & 0.4 & 0.4 & 0.4 & 0.5 & 0.4 & & & 0.4 \\
\hline Sig. & $* *$ & $* *$ & $* *$ & $* *$ & $* *$ & & & $* *$ \\
\hline
\end{tabular}

108,108 y $174 \%$ a las asociaciones $40: 40: 20,40: 30: 30$ y 40:20:40 (P $\leq$ 0.05). En verano la TC del pasto ovillo fue superior en 33, 129 y $200 \%$ a las de primavera, invierno y otoño $(P \leq 0.05)$. En ballico perenne la TC anual en la asociación 40:0:60 registró el valor más alto con $18 \mathrm{~kg} \mathrm{MS}$ ha $^{-1} \mathrm{~d}^{-1}$, mayor en 29,51 y $51 \%$ que en las asociaciones 40:30:30, 40:20:40 y 40:40:20, respectivamente. No hubo diferencias entre asociaciones en las TC de ballico perenne dentro de cada estación $(\mathrm{P}>0.05)$, excepto entre las asociaciones 40:0:60 y 40:40:20, que fueron las de mayor $y$ menor TC, respectivamente. Con excepción del otoño que mostró la menor TC (7 kg MS ha $\left.{ }^{-1} \mathrm{~d}^{-1}\right)$, no hubo diferencias $(\mathrm{P}>0.05)$ entre las demás estaciones del año.

Las TC del pasto ovillo y del ballico perenne aquí registradas son inferiores a las registradas en especies puras (Velasco et al., 2001, 2002), lo cual se atribuye a la competencia intra e interespecifica que se da entre especies por luz, agua y nutrientes, lo que reduce la tasa de aparición y elongación foliar en pastos y trébol blanco, como señalaron Durand et al. (1999).

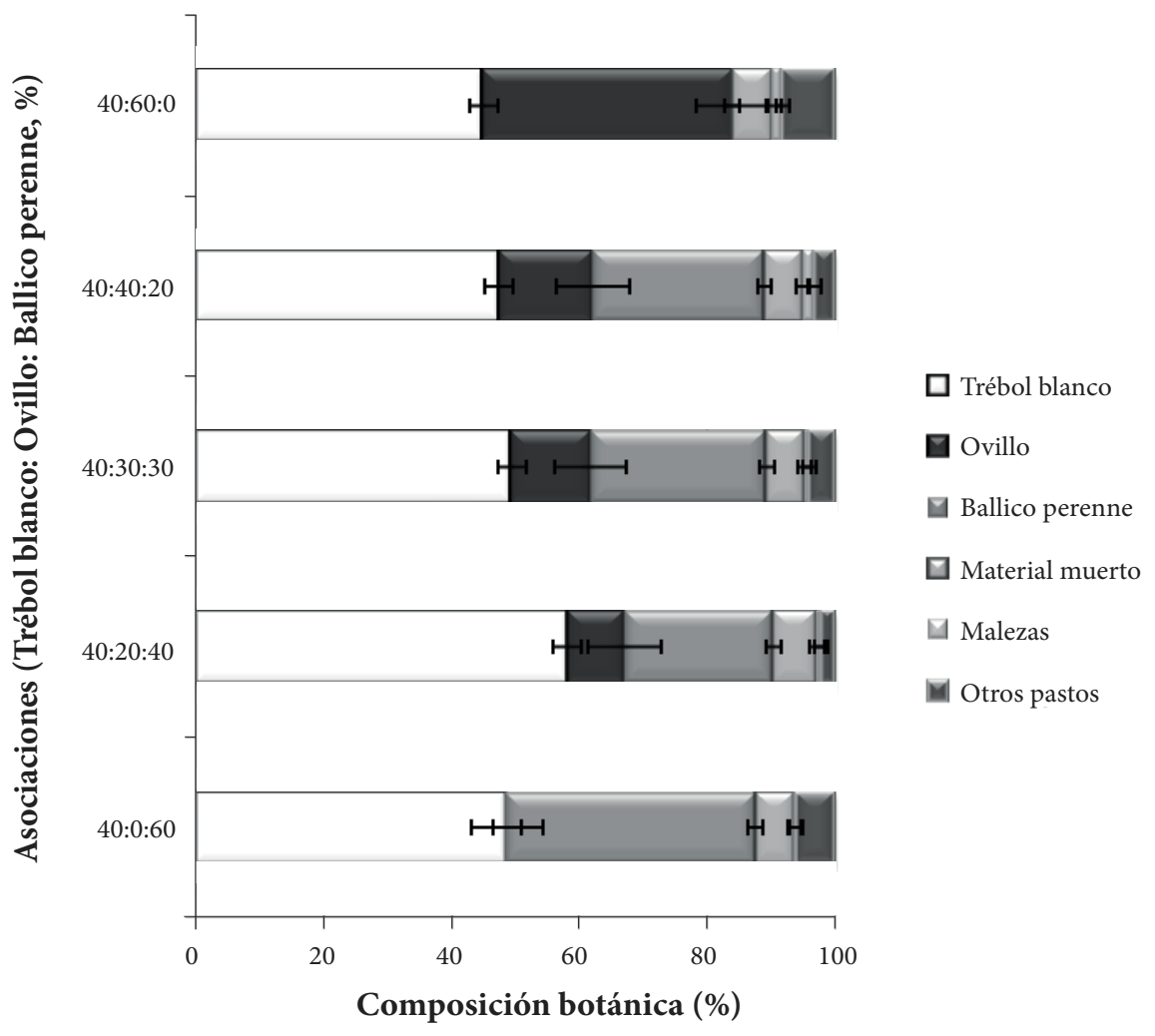

Figura 2. Porcentaje promedio de las especies deseables de cinco asociaciones de gramíneas con una leguminosa. 


\section{Altura de la pradera}

La altura de la asociación 40:30:30 superó a las demás asociaciones $(\mathrm{P} \leq 0.05)$. En verano se registró la mayor altura $(26 \mathrm{~cm})$, que superó en 17, 142 y $137 \%$ a las de primavera, otoño e invierno, respectivamente (Cuadro 5). En todas las épocas del año, la asociación 60:40:0 presentó las menores alturas. Lo contrario ocurrió con la asociación 40:30:30 que en todas las épocas superó a las demás asociaciones. Es decir, las alturas en las asociaciones estuvieron en relación directa con los rendimientos de materia seca. Según Castillo et al. (2009), la altura de la planta tiene relación positiva con la cantidad de forraje producido por la pradera, y el rendimiento de materia seca se puede estimar a partir de la altura medida antes del pastoreo. De acuerdo con Hodgson (1990), la altura de la pradera aunada a la densidad del forraje, determina la cantidad de forraje que se produce, mientras que la relación hoja:tallo y la diversidad entre especies determinan la calidad de la materia seca disponible.

\section{Composición botánica}

El trébol blanco contribuyó al rendimiento total de forraje con $50 \%$, el ballico perenne con $28 \%$ y el pasto ovillo con $12 \%$; el restante $10 \%$ lo integraron el material muerto, otros pastos y maleza (Figura 2). La contribución de cada especie al rendimiento de las asociaciones fue variable; el trébol blanco fue la especie que más contribuyó con valores que variaron de 58 a $42 \%$ en las asociaciones 40:20:40 y 40:60:0, respectivamente, con una diferencia de $4900 \mathrm{~kg} \mathrm{MS} \mathrm{ha-1} \mathrm{(Cuadro} \mathrm{2).} \mathrm{El} \mathrm{ballico} \mathrm{perenne} \mathrm{en} \mathrm{las}$ asociaciones 40:0:60 y 40:20:40 contribuyó con 36 y 23 \%, respectivamente (1990 $\mathrm{kg} \mathrm{MS} \mathrm{ha}^{-1}$, de diferencia). El pasto ovillo registró una diferencia de $2880 \mathrm{~kg} \mathrm{MS} \mathrm{ha}^{-1}$ entre las asociaciones 40:60:0 y 40:20:40.

La intensidad de pastoreo pudo ser el factor que permitió al trébol blanco ser la especie dominante durante todo el periodo experimental en las cinco asociaciones, probablemente porque su hábito de crecimiento estolonífero lo hace menos susceptible a perder meristemos de crecimiento durante el pastoreo, en comparación con las especies erectas como el ballico perenne y ovillo (Hodgson, 1990). Adicionalmente, se considera que el arreglo horizontal de sus hojas permitió a las plantas de trébol blanco restablecer su área foliar y utilizar la luz solar más rápido que el ballico perenne y ovillo (Brock et al., 1989). La asociación 40:20:40 fue la de mayor rendimiento de materia seca anual (Cuadro 1), donde el trébol aportó $58 \%$, seguido del ballico con $23 \%$, y del ovillo con $11 \%$. En las asociaciones de sólo dos especies (40:60:0 y 40:0:60) las aportaciones de material muerto, maleza y otros pastos, representaron más de $10 \%$ de la composición botánica, mientras que en las asociaciones de tres especies tal porcentaje fue menor a 10 (Figura 2).

\section{Análisis de mezclas}

Se fijó la proporción de trébol blanco en 40 \% de acuerdo con las recomendaciones de White y Hodgson (1999), y se aplicó la siguiente ecuación de regresión: Rendimiento de MS $\left(\mathrm{kg} \mathrm{ha}^{-1}\right)=11906$ (proporción de pasto ovillo) +14913 (proporción de ballico perenne) + 12770 (proporción de pasto ovillo) $\mathrm{x}$ (proporción de ballico perenne), obtenida en el análisis de mezclas. Los resultados indican que para obtener la asociación que maximiza el rendimiento de materia seca se deben incluir las siguientes proporciones: $40 \%$ de trébol blanco, $23 \%$ de pasto ovillo y $37 \%$ de ballico perenne.

\section{CONCLUSIONES}

La asociación 40:20:40 de trébol blanco-ovillo-ballico perenne presentó el mayor rendimiento anual y tasa de crecimiento promedio. Durante primavera-verano la asociación 40:20:40 de trébol blanco-ovillo-ballico perenne registró el mayor rendimiento de forraje y la mayor tasa de crecimiento, en tanto que en el otoño-invierno la asociación 40:30:30 observó el mayor rendimiento. El trébol blanco fue la especie dominante y contribuyó con $49 \%$ al rendimiento anual. De acuerdo con el análisis de mezclas, la combinación óptima para obtener el mayor rendimiento de forraje debe estar integrada por $40 \%$ de trébol blanco, $23 \%$ de pasto ovillo y $37 \%$ de ballico perenne.

\section{BIBLIOGRAFÍA}

Brock J L, J C Tilbrook (2000) Effect of cultivar of white clover on plant morphology during the establishment of mixed pastures under sheep grazing. New Zeal. J. Agric. Res. 43:335-343.

Brock J L, J R Caradus, M J M Hay (1989) Fifty years of white clover research in New Zealand. Proc. New Zeal. Grassland Assoc. 50:2539.

Camacho G J L, J G García M (2002) Producción y calidad del forraje de cuatro variedades de alfalfa asociadas con trébol blanco, ballico perenne, festuca alta y pasto ovillo. Vet. México. 34:149-177.

Castillo E G, B Valles M, J Jarillo R (2009) Relación entre materia seca presente y altura en gramas nativas del trópico mexicano. Téc. Pecu. Méx. 47:79-92.

Clark H, P C D Newton, C C Bell, E M Glasgow (1995) The influence of elevated $\mathrm{CO}_{2}$ and simulated seasonal changes in temperature on tissue turnover in pasture turfs dominated by ryegrass (Lolium perenne) and white clover (Trifolium repens). J. Appl. Ecol. 32:128136.

Durand J L, R Schäufele, F Gastal (1999) Grass leaf elongation rate as a function of developmental stage and temperature: Morphological analysis and modeling. Ann. Bot. 83:577-588.

García E (1988) Modificaciones al sistema de clasificación climática de Köppen. 4a ed. Universidad Nacional Autónoma de México. México, D. F. 217 p.

Hernández G A, J Hodgson, C Matthew (1997) Effect of spring grazing management on perennial ryegrass/white clover pastures. 1 . Tissue turnover and herbage accumulation. New Zeal. J. Agric. 
Res. 40:25-35

Hodgson J (1990) Grazing Management: Science into Practice. Longman Scientific and Technical. Harlow, England. 204 p.

Hodgson J, P N P Matthews, C Matthew, R J Lucas (1999) Pasture measurement. In: New Zealand Pasture and Crop Science. J White, J Hodgson (eds). Auckland, N. Z. Oxford University. pp:59-65.

Karsten H D, M Carlassare (2002) Describing the botanical compositions of a mixed species northeastern U. S. Pasture rotationally grazed by cattle. Crop Sci. 42:882-889.

Lemaire G (2001) Ecophysiology of grasslands: dynamics aspects of forage plant populations in grazed swards. In: Proc. XIX International Grassland Congress. Brazilian Society of Animal Husbandry, Sociedade Brasileira de Zootecnia (eds). São Pedro, São Paulo. Brazil. pp:29-37.

Matthew C G, E N Val Loo, E R Tom, L A Dawson, D A Care (2001) Understanding shoot and root development. In: Proc. XIX International Grassland Congress. Brazilian Society of Animal Husbandry, Sociedade Brasileira de Zootecnia (eds). São Pedro, São Paulo. Brazil. pp:19-27.

McKenzie B A, P D Kemp, D J Moot, C Matthew, R J Lucas (1999) Environmental effects on plant growth and development. In: New Zealand Pasture and Crop Science. J White, J Hodgson (eds). Auckland, N. Z. Oxford University. pp:29-44.

MINITAB (2006) Meet Minitab, Manual for the Basic Practice of Statistics. W H Freeman (ed). USA.

Ortiz S C (1997) Colección de Monolitos. Montecillo, Texcoco, Edo. México. México: Depto. Génesis de Suelos. Edafología, IRENAT. Colegio de Postgraduados. $17 \mathrm{p}$.

Quero C A R, J F Enríquez Q, L Miranda J (2007) Evaluación de especies forrajeras en América tropical, avances o status quo. Interciencia 32:566-571.
Sanderson M A, K J Soder, L D Muller, K D Klement, R H Skinner, S C Goslee (2005) Forage mixture productivity and botanical composition in pastures grazed by dairy cattle. Agron. J. 97:14651471.

SAS Institute (2002) SAS User's Guide: Statistics. 9th ed. Cary NC, USA SAS Inst. Inc.

Sevilla G A, A Pasinato, J M García (2001) Curvas de crecimiento de forrajeras templadas irrigadas. Arch. Latinoam. Animal Prod. 9:91-98.

Turner L R, D J Donaghy, P A Lane, R P Rawnsley (2006) Effect of defoliation interval on water-soluble carbohydrate and nitrogen energy reserves, regrowth of leaves and roots, and tiller number of cocksfoot (Dactylis glomerata L.) plants. Aust. J. Agric. Res. 57:243-249.

Velasco Z M E, A Hernández G, V A González H, J Pérez P, H Vaquera H, A Galvis S (2001) Curva de crecimiento y acumulación estaciona del pasto ovillo (Dactylis glomerata L.). Téc. Pecu. Méx. 39:1-14.

Velasco Z M E, A Hernández-Garay, H V A González, P J Pérez, H H Vaquera (2002) Curvas estacionales de crecimiento del ballico perenne. Rev. Fitotec. Mex. 25:97-106.

Velasco Z M E, A Hernández G, V A González H (2005) Rendimiento y valor nutritivo de ballico perenne (Lolium perenne L.) en respuesta a la frecuencia de corte. Téc. Pecu. Méx. 43:274-258.

White J, J Hodgson (1999) Pasture establishment. In: New Zealand Pasture and Crop Science. J White, J Hodgson (eds). Auckland, N. Z. Oxford University. pp:104-106

Zaragoza E J, A Hernández G, J Pérez P, J G Herrera H, F Osnaya G, P A Martínez H, S González M, A R Quero C (2009) Análisis de crecimiento estacional de una pradera asociada alfalfa-pasto ovillo. Téc. Pecu. Méx. 47:173-188. 